\title{
Van der Waals Coefficients for Nanostructures: Fullerenes Defy Conventional Wisdom
}

\author{
Adrienn Ruzsinszky, ${ }^{1}$ John P. Perdew, ${ }^{1}$ Jianmin Tao, ${ }^{1}$ Gábor I. Csonka, ${ }^{2}$ and J. M. Pitarke ${ }^{3,4}$ \\ ${ }^{1}$ Department of Physics and Engineering Physics, Tulane University, New Orleans, Louisiana 70118, USA \\ ${ }^{2}$ Department of Inorganic and Analytical Chemistry, Budapest University of Technology and Economics, \\ H-1521 Budapest, Hungary \\ ${ }^{3}$ CIC nanoGUNE Consolider, E-20018 Donostia-San Sebastian, Basque Country, Spain \\ ${ }^{4}$ Materia Kondentsatuaren Fisika Saila, DIPC, and Centro Fisica Materiales CSIC-UPV/EHU, \\ 644 Posta Kutxatila, E-48080 Bilbo, Basque Country, Spain
}

(Received 7 July 2012; published 5 December 2012)

\begin{abstract}
The van der Waals coefficients between quasispherical nanostructures can be modeled accurately and analytically by those of classical solid spheres (for nanoclusters) or spherical shells (for fullerenes) of uniform valence electron density, with the true static dipole polarizability. Here, we derive analytically and confirm numerically from this model the size dependencies of the van der Waals coefficients of all orders, showing, for example, that the asymptotic dependence for $C_{6}$ is the expected $n^{2}$ for pairs of nanoclusters $A_{n}-A_{n}$, each containing $n$ atoms, but $n^{2.75}$ for pairs of single-walled fullerenes $\mathrm{C}_{n}-\mathrm{C}_{n}$. Large fullerenes are argued to have much larger polarizabilities and dispersion coefficients than those predicted by either the standard atom pair-potential model or widely used nonlocal van der Waals correlation energy functionals.
\end{abstract}

DOI: 10.1103/PhysRevLett.109.233203

PACS numbers: $34.20 . \mathrm{Gj}, 36.40 .-\mathrm{c}, 68.65 .-\mathrm{k}$

The van der Waals attraction between two distant objects arises from correlations between density fluctuations on each, and can bind closed-shell objects. In particular, nanoclusters can form a molecular solid with a high sublimation point [1]. For two spherical objects $A$ and $B$ with centers separated by distance $d$, the nonretarded van der Waals interaction is [2]

$$
E_{\mathrm{vdW}}=-C_{6}^{A B} / d^{6}-C_{8}^{A B} / d^{8}-C_{10}^{A B} / d^{10}-\ldots
$$

The coefficients can be computed accurately from correlated wave functions only when the objects are atoms or small molecules. Standard semilocal density functionals for the exchange-correlation energy fail for the coefficients, predicting no interaction between nonoverlapped densities. This error is standardly corrected by using longrange additive pair potentials between atoms or effective atoms [3-6], or by using nonlocal correlation energy functionals developed to yield such a long-range interaction [7-11].

Recently, in an extension of our earlier work [12,13], we have found $[14,15]$ a way to estimate the van der Waals coefficients accurately between large quasispherical nanostructures, by modeling them as classical solid spheres or spherical shells of uniform valence electron density, with the outer radii fixed by accurate values for the static dipole polarizability of each nanostructure. Reference [15] presented the needed formulas for the coefficients of all orders, neglecting the energy gap except insofar as it affects the outer radius via Eq. (5) below. Reference [15] also discovered numerically that the size dependence of $C_{6}$ was the expected $n^{2}$ (the number of ways of pairing one atom in object $A$ with one in $B$ ) for solid nanocluster pairs
$A_{n}-A_{n}$, but showed a much stronger increase with the number of atoms $n$ for single-walled fullerene pairs $\mathrm{C}_{n}-\mathrm{C}_{n}$. Here we will derive the asymptotic $(n \rightarrow \infty)$ dependence of the van der Waals coefficients of all orders for both kinds of nanostructures, arguing that large fullerenes are unsuited to description either by atom pair potentials or by standard nonlocal van der Waals correlation energy functionals, and explaining why.

We begin with the second-order perturbation expression [2] for the van der Waals coefficient

$$
C_{2 k}^{A B}=\frac{(2 k-2) !}{2 \pi} \sum_{l_{1}=1}^{k-2} \frac{1}{\left(2 l_{1}\right) !\left(2 l_{2}\right) !} \int_{0}^{\infty} d u \alpha_{l_{1}}^{A}(i u) \alpha_{l_{2}}^{B}(i u),
$$

where $l_{2}=k-l_{1}-1$. Here, $\alpha_{l}^{A}(i u)$ is the $2^{l}$-pole dynamic polarizability (multipole linear response to an applied uniform electric field) of system $A$, evaluated at imaginary frequency iu ( $l=1$ dipole, 2 quadrupole, 3 octupole). Now consider a classical electrodynamic model, in which the $N$ valence electrons of the nanostructure are distributed with uniform density $\rho=N /\left\{(4 \pi / 3)\left[R^{3}-\right.\right.$ $\left.\left.(R-t)^{3}\right]\right\}$ over the spherical shell of inner radius $R-t$ and outer radius $R$. For a solid sphere, the shell thickness $t$ reduces to the outer radius $R$. The dielectric function is [16]

$$
\varepsilon(i u)=1+\omega_{p}^{2} /\left(\omega_{g}^{2}+u^{2}\right),
$$

where $\omega_{p}=\sqrt{4 \pi \rho}$ is the plasma frequency and $\omega_{g}$ is the energy gap. Equation (5) of Lucas et al. [17] with this $\varepsilon$ (and $\varepsilon_{i}=\varepsilon_{e}=1$ ) then gives the polarizability of this model system, 
TABLE I. Effect of the energy gap $\omega_{g}$ on the static multipole polarizabilities of a fullerene otherwise resembling $\mathrm{C}_{60}$, and on the van der Waals coefficients for a pair of such identical fullerenes, according to Eq. (4) and the Appendix. The outer radius is $R=8.11 \mathrm{bohr}$ and the shell thickness is $t=2.76 \mathrm{bohr}$ from Table 4. The calculated gap in the Kohn-Sham orbitalenergy spectrum for $\mathrm{C}_{60}$ is 0.0764 hartree $[18,19]$. (atomic units)

\begin{tabular}{lcccccc}
\hline \hline$\omega_{g}$ & $\frac{\alpha_{1}(0)}{10^{3}}$ & $\frac{\alpha_{2}(0)}{10^{5}}$ & $\frac{\alpha_{3}(0)}{10^{7}}$ & $\frac{C_{6}}{10^{3}(60)^{2}}$ & $\frac{C_{8}}{10^{5}(60)^{2}}$ & $\frac{C_{10}}{10^{7}(60)^{2}}$ \\
\hline 0 & 0.533 & 0.351 & 0.231 & 0.038 & 0.138 & 0.414 \\
0.0764 & 0.526 & 0.348 & 0.229 & 0.037 & 0.136 & 0.408 \\
0.382 & 0.497 & 0.335 & 0.222 & 0.034 & 0.127 & 0.385 \\
0.764 & 0.465 & 0.320 & 0.214 & 0.031 & 0.117 & 0.359 \\
7.64 & 0.220 & 0.182 & 0.130 & 0.010 & 0.045 & 0.150 \\
\hline \hline
\end{tabular}

$$
\alpha_{l}(i u)=R^{2 l+1} \frac{\omega_{l}^{2}}{\omega_{l}^{2}+\omega_{g}^{2}+u^{2}} \frac{1-\theta_{l}}{1-\beta_{l}(i u) \theta_{l}},
$$

where $\omega_{l}=\omega_{p} \sqrt{l /(2 l+1)}$ is the natural frequency of the $l$ th normal mode or surface plasmon in a solid metal sphere, $\theta_{l}=[(R-t) / R]^{2 l+1}=(1-t / R)^{2 l+1}$, and $\beta_{l}(i u)=\omega_{l}^{2} \tilde{\omega}_{l}^{2} /\left[\left(\omega_{l}^{2}+\omega_{g}^{2}+u^{2}\right)\left(\tilde{\omega}_{l}^{2}+\omega_{g}^{2}+u^{2}\right)\right]$. Here, $\tilde{\omega}_{l}=\omega_{p} \sqrt{(l+1) /(2 l+1)}$. The integration over $u$ in Eq. (2) can be done analytically, and the result is presented in the Appendix.

It is clear that the energy gap $\omega_{g}$ can be ignored (as it has been in our previous work and will be here) when $\omega_{g}^{2} \ll \omega_{p}^{2}$. Table I shows that even for the fullerene with the largest energy gap [16], $\mathrm{C}_{60}$, this is a valid approximation. Then the solid sphere or spherical shell is treated as metallic, $\beta_{l}(0)=1$, and the static dipole polarizability is just

$$
\alpha_{1}(0)=R^{3}(0<t \leq R) .
$$

Eq. (5) is independent of $t / R$ for a familiar classical reason from freshman physics: The equilibrium charge density on a conductor is distributed over its surface to create an induced field that cancels the external electric field inside the conductor. Because the van der Waals coefficients are sensitive to the static dipole polarizability, we employ the most sophisticated and realistic values of it that we can find to define the outer radius $R$. Then, for a single-wall fullerene with a known radius $R_{n}$ for the nuclear framework [18], we define the thickness $t$ as $2\left(R-R_{n}\right)$.

For a variety of real nanoclusters and fullerenes (Tables II and III, S2), this model predicts $C_{6}$ coefficients that agree well (mean absolute relative error about $7 \%$ [15]) with the best reference values we can find (typically time-dependent density functional or Hartree-Fock values for $\mathrm{Na}_{n}-\mathrm{Na}_{n}, \mathrm{Na}_{n}-\mathrm{C}_{60}$, and $\left.\mathrm{C}_{60}-\mathrm{C}_{60}\right)$. It also predicts higher-order van der Waals coefficients which we believe are realistic. For example, it predicts $C_{8}$ for a pair of $\mathrm{C}_{60}$ fullerenes within $8 \%$ [15] of a reference value [19]. [Higher accuracy, e.g., $3 \%$ for atom pairs, seems attainable
TABLE II. Model radius $R=\alpha_{1}(0)^{1 / 3}$ and van der Waals coefficients for $\mathrm{Na}_{n}-\mathrm{Na}_{n}$, in atomic units, demonstrating the predicted asymptotic behavior of Eq. (7). One valence electron per atom. The realistic reference value for $\alpha_{1}(0)$ and the coefficients $C_{6}, C_{8}$, and $C_{10}$ (from the Appendix with $\omega_{g}=0$ ) are from Ref. [15]. From $n=2$ to $92, C_{6}$ increases by a factor of more than 1000 , and $C_{10}$ by more than 150,000 .

\begin{tabular}{lcccc}
\hline \hline$n$ & $R$ & $\frac{C_{6}}{10^{3} n^{2}}$ & $\frac{C_{8}}{10^{5} n^{8 / 3}}$ & $\frac{C_{10}}{10^{7} n^{10 / 3}}$ \\
\hline 2 & 6.38 & 1.11 & 1.49 & 1.65 \\
4 & 8.00 & 1.08 & 1.44 & 1.59 \\
6 & 9.06 & 1.04 & 1.34 & 1.45 \\
8 & 9.60 & 0.87 & 1.05 & 1.05 \\
10 & 10.17 & 0.81 & 0.95 & 0.91 \\
12 & 11.03 & 0.89 & 1.08 & 1.08 \\
14 & 11.82 & 0.96 & 1.21 & 1.26 \\
18 & 11.99 & 0.70 & 0.77 & 0.70 \\
20 & 12.57 & 0.74 & 0.83 & 0.78 \\
40 & 15.18 & 0.61 & 0.63 & 0.54 \\
58 & 16.98 & 0.58 & 0.59 & 0.49 \\
92 & 19.56 & 0.55 & 0.54 & 0.44 \\
\hline \hline
\end{tabular}

from formulas $[12,13,15]$ that employ a more-realistic radially varying density $\rho(r)$.]

To understand how this model can be accurate, even when the real system has an energy gap and a highly nonuniform density (and even for atoms [15]), consider Eq. (2) for $C_{6}(k=3)$. The model smoothly interpolates the dynamic dipole polarizability $\alpha_{1}(i u)$ from its exact static $(u=0)$ limit to zero $(u \rightarrow \infty)$, in a way that is exact

TABLE III. Model outer radius $R=\alpha_{1}(0)^{1 / 3}$, shell thickness $t=2\left(R-R_{n}\right)$, and van der Waals coefficients for single-walled fullerene pairs $\mathrm{C}_{n}-\mathrm{C}_{n}$, in atomic units, demonstrating the predicted asymptotic behavior of Eq. (8). Four valence electrons per atom. The realistic reference values for $\alpha_{1}(0)$ and the coefficients $C_{6}, C_{8}$, and $C_{10}$ for $n \leq 60$ are from Ref. [15]. For $n>60$, see the explanation in the second paragraph following Eq. (10). From $n=20$ to $3840, C_{6}$ increases by a factor of more than 394000 , and $C_{10}$ by more than $5.5 \times 10^{9}$. Note that the asymptotic limit $(n \rightarrow \infty)$ is reached slowly when $t / R$ differs from 1 . To the extent that the larger fullerences are nonspherical (e.g., icosahedral in $\mathrm{C}_{540}$ ) or have an unusual band structure [24], the predictions in this table may deviate from realism, but they suffice to establish in principle the failure of the atom pairinteraction model, in which $C_{6} \sim n^{2}$ as $n \rightarrow \infty$.

\begin{tabular}{lrcccc}
\hline \hline$n$ & $R$ & $t$ & $\frac{C_{6}}{n^{11 / 4}}$ & $\frac{C_{8}}{10 n^{15 / 4}}$ & $\frac{C_{10}}{100 n^{19 / 4}}$ \\
\hline 20 & 5.13 & 2.40 & 2.75 & 1.98 & 1.14 \\
60 & 8.11 & 2.76 & 1.75 & 1.07 & 0.53 \\
240 & 14.46 & 2.76 & 1.00 & 0.37 & 0.20 \\
540 & 20.88 & 2.76 & 0.82 & 0.23 & 0.14 \\
960 & 26.91 & 2.76 & 0.70 & 0.16 & 0.11 \\
2160 & 40.12 & 2.76 & 0.67 & 0.11 & 0.10 \\
3840 & 51.95 & 2.76 & 0.57 & 0.08 & 0.09 \\
\hline \hline
\end{tabular}


for a classical metallic spherical shell and useful for many other densities. (For the solid-sphere $u$ dependence, see figures in Ref. [13].)

What is the asymptotic $(n \rightarrow \infty)$ behavior of the coefficient $C_{2 k}^{A A}$ between two identical quasispherical nanostructures, each containing $n$ atoms? We may expect that the density $\rho$ tends to a constant in this limit, as does the thickness $t$ of a single-walled fullerene. (In fact, we find only weak variation of the density from the smallest to the largest clusters of a given chemical element.) Then the asymptotic behavior is controlled by $R$, and by simple geometry

$$
n \sim R^{\delta},
$$

where $\delta$ is the dimensionality ( 3 for a solid sphere where the atoms are distributed over the three-dimensional interior, or 2 for a single-walled fullerene where the atoms are distributed over the two-dimensional surface of the sphere). If we ignore the dependence of $\theta_{l}$ upon $R$ (as we can for a solid sphere where $\theta_{l}=0$ for all $R$ ), we easily find from Eqs. (2) and (4) that

$$
C_{2 k}^{A A} \sim R^{2 k} \sim n^{2 k / \delta}(\delta=3) .
$$

In particular, Eq. (7) makes $C_{6}^{A A} \sim n^{2}$ for $\delta=3$ (solid spheres), but $C_{6}^{A A} \sim n^{3}$ for $\delta=2$ (fullerenes). The atom pair-potential picture predicts $n^{2}$ for both, since $n^{2}$ is the number of ways to pair an atom in the first nanostructure with one in the second. Within the atom pair-potential model, this $n^{2}$ dependence applies only for $C_{6}$, because $r_{i j}^{-6}$ equals $d^{-6}$ plus higher-order corrections that contribute to $C_{8}$ and higher-order coefficients. We will show elsewhere [20] that the pair interaction model of Ref. [3] predicts $C_{8} \sim n^{8 / 3}$ and $C_{10} \sim n^{10 / 3}$, also in agreement with Eq. (7) for $\delta=3$.

The simple analysis leading to Eq. (7) is not quite right for a single-walled fullerene, for which $\theta_{l} \rightarrow$ $1-(2 l+1) t / R$ as $R \rightarrow \infty$. In this case, we have to revert to the equations in the Appendix, which show that

$$
\begin{aligned}
& C_{2 k}^{A A} \sim R^{2 k}(t / R)^{2} /(t / R)^{3 / 2} \sim R^{2 k-1 / 2} \sim n^{(2 k-1 / 2) / \delta} \\
& \quad(\delta=2, \text { metallic })
\end{aligned}
$$

Then $C_{6}^{A A} \sim n^{2.75}$ for a pair of identical single-walled fullerenes. All of the van der Waals coefficients between identical single-walled fullerenes grow much faster with $n$ than the atom pair-potential model predicts. For example, if we use the same $C_{6}$ for a pair of bound carbon atoms that was used in Ref. [6], we find $C_{6}$ for $\mathrm{C}_{20}-\mathrm{C}_{20}$ to be $(20)^{2}(26.4)=10500(10400)$, for $\mathrm{C}_{60}-\mathrm{C}_{60}(60)^{2}(26.4)=$ $94900(137000)$, and for $\mathrm{C}_{3840}-\mathrm{C}_{3840}(3840)^{2}(26.4)=$ $3.9 \times 10^{8}\left(4.1 \times 10^{9}\right)$ (where the values in parentheses are accurate ones from our spherical-shell model [15]). The simple explanation is that the fullerene is essentially metallic with all its atoms distributed abnormally far from its center, so these atoms can make a larger static dipole polarizability [Eq. (5)] and van der Waals interaction than they could if distributed with the same density over a solid sphere.

The fullerenes have an energy gap $\omega_{g}$ that tends to zero as $n \rightarrow \infty$ and the surface tends to graphene. In fact, as discussed in the Appendix, it seems that

$$
\lim _{n \rightarrow \infty}(2 / 3)\left(\omega_{g} / \omega_{p}\right)^{2} /(t / R)=0
$$

for the fullerenes. If Eq. (9) did not hold, we would have

$$
\begin{aligned}
& C_{2 k}^{A A} \sim R^{2 k}(t / R)^{2} \sim R^{2 k-2} \sim n^{(2 k-2) / \delta} \\
& \quad(\delta=2, \text { nonmetallic }) .
\end{aligned}
$$

Eq. (10) would predict the normal behavior $C_{6}^{A A} \sim n^{2}$.

Tables II and III and S1 [21] show that our analytic asymptotic behaviors are nicely confirmed for $C_{6}, C_{8}$, and $C_{10}$ by our model calculations for the solid-sphere clusters $\mathrm{Na}_{n}-\mathrm{Na}_{n}$ and $\mathrm{Si}_{n}-\mathrm{Si}_{n}$ and the hollow-sphere single-walled fullerene clusters $\mathrm{C}_{n}-\mathrm{C}_{n}$. Most of the input used to construct these tables was taken from Ref. [15], which also lists the sources for the accurate reference values.

In our earlier work [15], we took the static dipole polarizabilities $\alpha_{1}(0)$ for the larger fullerenes $\mathrm{C}_{n}(n>60)$ from a tight-binding model $[18,19]$. But we were suspicious of these values, because they made the thickness $t$ increase from the reasonable $t=2.76$ bohr for $n=60$ to the unreasonably large $t=20.97 \mathrm{bohr}$ for $n=3840$. Here we have instead fixed $t=2.76$ bohr for all $n \geq 60$, which leads to a static dipole polarizability $\left(R_{n}+t / 2\right)^{3}$ for $n=3840$ that is only $60 \%$ of that predicted by the tight-binding model. We believe that our revised static polarizabilities for the larger fullerenes are essentially correct.

We can also predict and confirm the asymptotic size dependence of $C_{2 k}^{A B}$ for $\mathrm{Na}_{n}-\mathrm{C}_{60}$. The highest contributing power of $R_{\mathrm{Na}}$ is $\left(R_{\mathrm{Na}}\right)^{2 k-3}$, so $C_{2 k} \sim n^{(2 k-3) / 3}$ (Table S2 [21]).

The larger single-walled fullerenes pose a challenge not only to the atom pair-potential model but also to a nonlocal van der Waals correlation energy functional of the form [7-11]

$$
E_{c}[n]=\int d^{3} r \int d^{3} r^{\prime} g\left(n(\vec{r}), \nabla n(\vec{r}) ;\left|\vec{r}^{\prime}-\vec{r}\right| ; n\left(\vec{r}^{\prime}\right), \nabla n\left(\vec{r}^{\prime}\right)\right) .
$$

These functionals are designed to produce the leading term of Eq. (1), but not necessarily with the correct value for the coefficient $C_{6}^{A B}$. By comparison with Eq. (2), it is clear that these functionals make a generalized gradient approximation for the static dipole polarizability

$$
\alpha_{1}^{A}(0)=\int d^{3} r h_{A}[n(\vec{r}), \nabla n(\vec{r})] .
$$

Vydrov and Van Voorhis [22] have extracted the corresponding $C_{6}^{A B}$ coefficients between small molecules for several of the van der Waals correlation functionals. The 
Langreth-Lundqvist 2004 (vdW-DF) [7] has a mean absolute relative error of $20 \%$, which increases to $60 \%$ (with all coefficients underestimated) in the Langreth-Lundqvist 2010 (vdW-DF2) [8]. Their own Vydrov-Van Voorhis 2009 [9] and 2010 [10] functionals both have the same $C_{6}^{A B}$, with a mean absolute relative error of only $10 \%$. But it is clear that Eq. (12) can be right for solid spheres only by being wrong for hollow conducting spheres. If we regard our sharply cutoff densities as limits of smoother exponentially decaying densities, we easily find that the VydrovVan Voorhis "local polarizability model" [22] of the form of Eq. (12) predicts for a spherical shell

$$
\alpha_{1}^{V V}(0)=R^{3}-(R-t)^{3} .
$$

At constant density, Eq. (13) is proportional to the number of atoms $n$ and thus consistent with the atom pair-potential model. Comparison with Eq. (5) shows that Eq. (13) is correct for a solid sphere $(t=R)$, but not for a hollow conducting sphere, where it predicts a static dipole polarizability smaller than the exact $R^{3}\left(0.85 R^{3}\right.$ for $\mathrm{C}_{20}$ and $0.71 R^{3}$ for $\mathrm{C}_{60}$, etc.). The polarizability itself can be a radically nonlocal functional of the electron density. This effect is captured by our present approach, by the random phase approximation $[23,24]$, and by time-dependent density functional theory. A nonlocal polarizability model is also employed, at low computational cost, in Ref. [25], where it was found to work well for normal molecules and clusters.

To confirm the conclusions of the preceding paragraph, we have used the Vydrov-Van Voorhis 2010 functional to evaluate the $C_{6}$ coefficients for $\mathrm{Na}_{20}-\mathrm{Na}_{20}, \mathrm{C}_{20}-\mathrm{C}_{20}$, and $\mathrm{C}_{60}-\mathrm{C}_{60}$. For spherical shells, Eq. (14) of Ref. [22] has been evaluated exactly by solid geometry: $C_{6}^{V V 10}=$ $0.006766 \omega_{p}\left[(2 R)^{3}-(2 R-2 t)^{3}\right]^{2}$. The results (in comparison to accurate values from Ref. [15]) are respectively 297000 (297 000), 8200 (10 400), and 86200 (137 000) atomic units. Moreover, the accurate values cited here are evaluated for the same model conducting spherical-shell density to which we have applied the VV10 functional. Thus, we have identified a fundamental limitation to the improvement of functionals with the form of Eq. (11).

Dobson and collaborators [24,26,27] argued that pairinteraction models and functionals of the form of Eq. (11) fail for interactions between zero-gap systems that are extended in at least one spatial dimension (so that electrons can flow over large distances) but are constricted in another dimension (so that the screening is reduced compared to that in bulk 3D metals). They considered specifically onedimensional and planar two-dimensional systems, but a more significant difference between their work and ours lies in the order of limits. They considered the case in which a large dimension of the system tends to infinity before the separation between systems does, leading to an unexpected asymptotic dependence of the interaction upon separation. We consider the case in which the separation goes to infinity first, so that the asymptotic dependence on separation is the expected one but the van der Waals coefficients grow with the large dimension of the system in an unexpected way. In either case, the van der Waals interaction is stronger than expected. The case of Dobson and collaborators is most relevant to condensed matter physics, while our case is most relevant to chemistry. (For a third case, consider an atom interacting with a distant but fully extended metal surface, as discussed by Cole et al. [28].)

In separate work [20], we have used the exact $C_{2 k}^{A A}$ for our classical metallic spherical shell to show that the van der Waals series can be summed geometrically and that the resulting divergence at $d=2 R$ can be removed by an exponentially-decaying correction which does not change the asymptotic series to any order in $d^{-1}$. In that work, we have also extracted the size dependence of the van der Waals coefficients of all orders as predicted by the pairinteraction model of Ref. [3].

In summary, the van der Waals coefficients $C_{6}^{A B}$ between solid quasispherical nanoclusters are normal, and can be predicted by effective-atom pair potentials or by nonlocal correlation energy functionals of the form of Eq. (9). But the van der Waals coefficients of all orders between singlewalled fullerenes are abnormally large, because of the abnormal distribution of atoms away from the centers and the asymptotic metallicity, and cannot be so described.

This work was supported in part by the National Science Foundation under Grant No. DMR-0854769 and Cooperative Agreement No. EPS-1003897, with additional support from the Louisiana Board of Regents.

\section{APPENDIX}

Detailed expressions for the van der Waals coefficients from Eqs. (2) and (4).

$$
\begin{gathered}
C_{2 k}^{A B}=\frac{(2 k-2) !}{2 \pi} \sum_{l_{1}=1}^{k-2} \frac{R_{A}^{2 l_{1}+1}}{\left(2 l_{1}\right) !} \frac{R_{B}^{2 l_{2}+1}}{\left(2 l_{2}\right) !} \\
\times\left(\omega_{l_{1}}^{A}\right)^{2}\left(\omega_{l_{2}}^{B}\right)^{2}\left(1-\theta_{l_{1}}^{A}\right)\left(1-\theta_{l_{2}}^{B}\right) I_{l_{1}, l_{2}}^{A B} . \\
I_{l_{1}, l_{2}}^{A B}=\frac{\pi}{2 Q_{l_{1}, l_{2}}^{A B}} \sum_{i=1}^{3}\left[\frac{f_{i}\left(a_{l_{1}}^{A}, a_{l_{2}}^{B} ; b_{l_{1}}^{A}, b_{l_{2}}^{B}\right)}{D\left(a_{l_{1}}^{A}, a_{l_{2}}^{B}\right)}+\frac{f_{i}\left(b_{l_{1}}^{A}, b_{l_{2}}^{B} ; a_{l_{1}}^{A}, a_{l_{2}}^{B}\right)}{D\left(b_{l_{1}}^{A}, b_{l_{2}}^{B}\right)}\right] . \\
Q_{l_{1}, l_{2}}^{A B}=\left[\left(a_{l_{1}}^{A}\right)^{2}-\left(b_{l_{1}}^{A}\right)^{2}\right]\left[\left(a_{l_{1}}^{A}\right)^{2}-\left(b_{l_{2}}^{B}\right)^{2}\right] \\
\quad \times\left[\left(a_{l_{2}}^{B}\right)^{2}-\left(b_{l_{1}}^{A}\right)^{2}\right]\left[\left(a_{l_{2}}^{B}\right)^{2}-\left(b_{l_{2}}^{B}\right)^{2}\right] . \\
D(x, y)=x y(x+y) . \\
a_{l}=\frac{1}{\sqrt{2}}\left[\left(\omega_{l}^{2}+\tilde{\omega}_{l}^{2}+2 \omega_{g}^{2}\right)\right. \\
\left.-\sqrt{\left(\omega_{l}^{2}-\tilde{\omega}_{l}^{2}\right)^{2}+4 \theta_{l} \omega_{l}^{2} \tilde{\omega}_{l}^{2}}\right]^{1 / 2} .
\end{gathered}
$$




$$
\begin{aligned}
b_{l}= & \frac{1}{\sqrt{2}}\left[\left(\omega_{l}^{2}+\tilde{\omega}_{l}^{2}+2 \omega_{g}^{2}\right)\right. \\
& \left.+\sqrt{\left(\omega_{l}^{2}-\tilde{\omega}_{l}^{2}\right)^{2}+4 \theta_{l} \omega_{l}^{2} \tilde{\omega}_{l}^{2}}\right]^{1 / 2} \\
f_{1}(p, q ; s, t)=(p q)^{4}+(p q)^{3}\left(s^{2}+t^{2}\right) & \quad-p q(s t)^{2}\left(p^{2}+p q+q^{2}\right) . \\
f_{2}(p, q ; s, t)=- & \left\{\left(\tilde{\omega}_{l_{1}}^{A}\right)^{2}+\left(\omega_{g}^{A}\right)^{2}+\left(\tilde{\omega}_{l_{2}}^{B}\right)^{2}+\left(\omega_{g}^{B}\right)^{2}\right\} \\
\times & {\left[(p q)^{2}\left(p^{2}+p q+q^{2}-s^{2}-t^{2}\right)-p q(s t)^{2}\right] . } \\
f_{3}(p, q ; s, t)= & {\left[\left(\tilde{\omega}_{l_{1}}^{A}\right)^{2}+\left(\omega_{g}^{A}\right)^{2}\right]\left[\left(\tilde{\omega}_{l_{2}}^{B}\right)^{2}+\left(\omega_{g}^{B}\right)^{2}\right] } \\
& \times\left[p^{4}+p^{3} q+p^{2} q^{2}+p q^{3}+q^{4}\right. \\
& \left.-\left(p^{2}+p q+q^{2}\right)\left(s^{2}+t^{2}\right)+(s t)^{2}\right]
\end{aligned}
$$

The corresponding expressions for $\omega_{g}=0$ were presented in Ref. [15]. Now consider $A=B$. For a solid sphere, $\theta_{l}=0$ and all the asymptotic size dependence is in the factor $R^{2 k}$. For a single-walled fullerene, the energy gap $\omega_{g}$ vanishes asymptotically as the fullerene shell becomes graphene, and $\theta_{l}$ tends to 1 . The factor $\left(1-\theta_{l_{1}}\right)\left(1-\theta_{l_{2}}\right)$ is asymptotically proportional to $(t / R)^{2} . a_{l}$ is asymptotically proportional to $(t / R)^{1 / 2}$, and $D\left(a_{l_{1}}, a_{l_{2}}\right)$ to $(t / R)^{3 / 2}$. Hence, Eq. (8).

To be very careful about the asymptotics, we should also consider the size dependence of the band gap $\omega_{g}$, which has been calculated for $\mathrm{C}_{n}$ in Ref. [19]. Using those values, we find that $(2 / 3)\left(\omega_{g} / \omega_{p}\right)^{2} /(t / R)$ is very small and deceases monotonically from $n=60$ to $n=3840$, suggesting that $a_{l}$ does indeed behave asymptotically like $(t / R)^{1 / 2}$.

[1] R. Loutfy and E. Wexler, in Perspectives of Fullerene Nanotechnology, Part VII, edited by E. Osawa (Kluwer, New York, 2002).

[2] H. B. G. Casimir and D. Polder, Phys. Rev. 73, 360 (1948).

[3] H. C. Hamaker, Physica (Amsterdam) 4, 1058 (1937).

[4] A. Tkatchenko and M. Scheffler, Phys. Rev. Lett. 102, 073005 (2009).
[5] S. Grimme, Wiley Interdiscip. Rev. Comput. Mol. Sci. 1, 211 (2011).

[6] S. N. Steinmann, G. I. Csonka, and C. Corminboeuf, J. Chem. Theory Comput. 5, 2950 (2009).

[7] M. Dion, H. Rydberg, E. Schröder, D. C. Langreth, and B. I. Lundqvist, Phys. Rev. Lett. 92, 246401 (2004).

[8] K. Lee, E. D. Murray, L. Kong, B. I. Lundqvist, and D. C. Langreth, Phys. Rev. B 82, 081101 (2010).

[9] O. A. Vydrov and T. Van Voorhis, Phys. Rev. Lett. 103, 063004 (2009).

[10] O. A. Vydrov and T. Van Voorhis, J. Chem. Phys. 133, 244103 (2010).

[11] G. Roman-Perez and J.M. Soler, Phys. Rev. Lett. 103, 096102 (2009).

[12] J. Tao, J. P. Perdew, and A. Ruzsinszky, Phys. Rev. B 81, 233102 (2010).

[13] J. Tao, J. P. Perdew, and A. Ruzsinszky, Proc. Natl. Acad. Sci. U.S.A. 109, 18 (2012).

[14] J. P. Perdew, J. Tao, P. Hao, A. Ruzsinszky, G. I. Csonka, and J. M. Pitarke, J. Phys. Condens. Matter 24, 424207 (2012).

[15] J. Tao and J.P. Perdew (to be published).

[16] Z. H. Levine and S. G. Louie, Phys. Rev. B 25, 6310 (1982).

[17] A. A. Lucas, L. Henrard, and Ph. Lambin, Phys. Rev. B 49, 2888 (1994).

[18] G. K. Gueorguiev, J. M. Pacheco, and D. Tomanek, Phys. Rev. Lett. 92, 215501 (2004).

[19] J. M. Pacheco and J.P. P. Ramalho, Phys. Rev. Lett. 79, 3873 (1997).

[20] J. P. Perdew, A. Ruzsinszky, J. Sun, S. Glindmeyer, and G. I. Csonka (to be published).

[21] See Supplemental Material at http://link.aps.org/ supplemental/10.1103/PhysRevLett.109.233203 for the analogs of Tables I, II, and III for $\mathrm{Si}_{n}-\mathrm{Si}_{n}$ and $\mathrm{Na}_{n}-\mathrm{C}_{60}$.

[22] O. A. Vydrov and T. Van Voorhis, Phys. Rev. A 81, 062708 (2010).

[23] D. C. Langreth and J. P. Perdew, Phys. Rev. B 21, 5469 (1980).

[24] J. F. Dobson, A. White, and A. Rubio, Phys. Rev. Lett. 96, 073201 (2006).

[25] A. Tkatchenko, R. A. DiStasio, R. Car, and M. Scheffler, Phys. Rev. Lett. 108, 236402 (2012).

[26] A. White and J.F. Dobson, Phys. Rev. B 77, 075436 (2008).

[27] R.-F. Liu, J. G. Ángyán, and J. F. Dobson, J. Chem. Phys. 134, 114106 (2011).

[28] M. W. Cole, D. Velegol, H.-Y. Kim, and A. A. Lucas, Mol. Simul. 35, 849 (2009). 